\title{
Sleep disorders in attention-deficit hyperactivity disorder and autism spectrum disorder: a pragmatic approach to assessment and management
}

Paul Shanahan is a behaviour analyst and research lead. He practises in a neurodevelopmental service for Your Healthcare Community Interest Company (CIC), London, UK. $\mathrm{He}$ is also a postgraduate researcher at the University of Surrey and has a range of interests, including healthcare delivery and sleep disorders. Miriam Isaac is a consultant psychiatrist in psychiatry of intellectual disabilities. She is currently working with the National Deaf Child and Adolescent Mental Health Service (CAMHS) in York, UK, and leads the sleep pathway for the Northern Arm of the National Deaf CAMHS. Her specialist clinical interests are in sleep psychiatry and neurodevelopmental disorders. Jane Blackwell is a research fellow working in the Child Oriented Mental Health Intervention Centre (COMIC) at the University of York, UK, as the trial coordinator for the Autism Spectrum Social Stories ${ }^{\mathrm{TM}}$ In Schools Trial 2. She also coordinates the White Rose Child and Adolescent Sleep Research Network. Her doctoral and postdoctoral research investigated the relationship between sleep, physical activity, cognitive function and psychosocial well-being in children with narcolepsy.

Correspondence Paul J. Shanahan. Email: p.j.shanahan@surrey.ac.uk

First received 17 Apr 2020

Final revision 27 Jul 2020

Accepted 5 Aug 2020

\section{Copyright and usage}

(C) The Author(s) 2020. Published by Cambridge University Press on behalf of the Royal College of Psychiatrists. This is an Open Access article, distributed under the terms of the Creative Commons Attribution licence (http://creativecommons.org/ licenses/by/4.0/), which permits unrestricted re-use, distribution, and reproduction in any medium, provided the original work is properly cited.

\author{
Paul J. Shanahan (, Miriam Isaac \& Jane E. Blackwell
}

\section{SUMMARY}

Sleep is essential for survival and humans spend approximately one-third of their life asleep. Adequate sleep is needed to maintain both physical and psychological health. Routinely getting less than the recommended amount of sleep for your age can have profound negative effects on health, such as increasing the likeliness of psychiatric illness, diabetes, cardiovascular disease and stroke. In children and adults with neurodevelopmental disorders, the prevalence of sleep disorders is significantly higher than in the general population. Given the relationship between sleep and psychiatric disorders, it is essential that psychiatrists have knowledge of the principles of sleep medicine. In this article, we focus on the common sleep disorders found in those with attention-deficit hyperactivity disorder (ADHD) and autism spectrum disorder (ASD) and give an overview of screening, diagnosis and management.

\section{LEARNING OBJECTIVES}

After reading this article you will be able to:

- recognise the common coexisting sleep disorders in individuals with ASD and ADHD

- develop a working knowledge of sleep disorders that may be encountered in psychiatric practice (with particular focus on individuals with ADHD and ASD)

- demonstrate a broad understanding of how sleep disorders are investigated and treated in children, adolescents and adults with ADHD and ASD.

\section{KEYWORDS}

Attention-deficit hyperactivity disorders; autism spectrum disorders; insomnia; circadian rhythm sleep-wake disorders; parasomnias.

\section{Fundamentals of normal sleep}

Sleep is a fundamental biological function and on average we spend about one-third of our life asleep (Stores 2009). Disruptions to a restorative sleep can affect an individual's neurocognition, mood and daytime functioning (Wajszilber 2018). Longterm sleep disturbance can result in broad health consequences, including coronary heart disease, stroke, inflammatory disease and increased likelihood of type 2 diabetes onset (Cappuccio 2010, 2011; Irwin 2016).

\section{Normal sleep and wakefulness}

There are two types of sleep: non-rapid eye movement (NREM) and rapid eye movement (REM) sleep. The stages of sleep are characterised by different patterns of brain activity, eye movements and muscle tone. The basic structure of sleep is referred to as 'sleep architecture' and can be represented using a hypnogram (Fig. 1). Typical overnight sleep usually begins with NREM sleep and then follows a cyclical pattern alternating between NREM and REM sleep phases, with brief wakings every $90 \mathrm{~min}$ until the person wakes up.

The sleep-wake cycle is one of the circadian rhythms and runs roughly every $24 \mathrm{~h}$. These rhythms are generated by the suprachiasmatic nucleus in the hypothalamus, which functions as a biological clock (Dunlap 2004). The sleep-wake system is thought to be regulated by two major processes, one that drives sleep (process S) and one that maintains wakefulness (process C) (Gillette 2005). The need for sleep (process S) accumulates across the day, peaking just before bedtime. This homeostatic drive for sleep is reduced following adequate rest at night and the circadian waking drive (process C) begins to increase, and then the cycle starts again. 


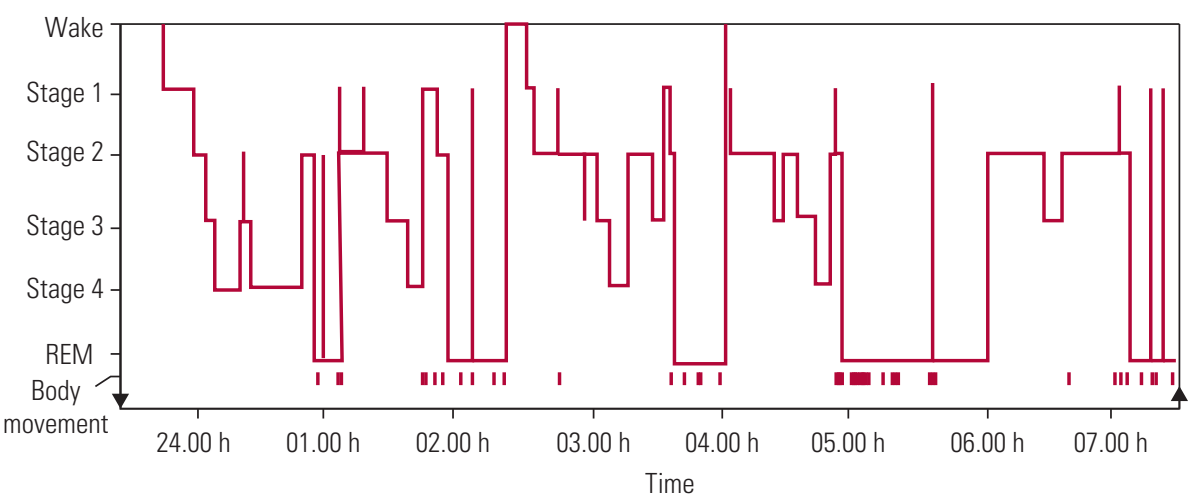

FIG 1 Progression of sleep states across a single night in a young adult. REM, rapid eye movement (Carskadon 2005). (C) 2006 National Academy of Sciences: reproduced with permission of Elsevier.

As shown in Fig. 2, there are normal variations with age in how sleep is initiated and maintained, the percentage of time spent in each stage of sleep and overall sleep efficiency (Altevogt 2006).

Restorative sleep is important in learning, memory and affect regulation. Sleep deprivation has been shown to have a negative impact on learning, and research suggests that sleep has an important role in regulating the synaptic plasticity and connectivity of the brain. Together with impairments of attention and alertness, sleep deprivation is commonly associated with increased subjective reports of irritability and mood changes (Walker 2009).

\section{Diagnostic classification of sleep disorders}

The International Classification of Sleep Disorders, now in its third edition (ICSD-3), can be used to diagnose insomnia, sleep-related breathing disorders, central disorders of hypersomnolence, circadian rhythm sleep-wake disorders, parasomnias and sleep-related movement disorders such as restless legs syndrome, periodic limb movement disorder and sleep-related leg cramps (American Academy of Sleep Medicine 2014).

The two most commonly reported sleep disorders in individuals with attention-deficit hyperactivity disorder (ADHD) or autism spectrum disorder (ASD) are insomnia and circadian rhythm sleepwake disorders (ICD-10 and DSM-5 'circadian rhythm sleep disorders NOS'). Insomnia is difficulty getting to sleep or staying asleep despite having opportunity and circumstances to sleep, leading to unsatisfactory sleep or daytime consequences (American Academy of Sleep Medicine 2014; Selsick 2018). To be classified as having insomnia the individual must present with these difficulties for at least 3 months and the problems must occur at least three times a week.

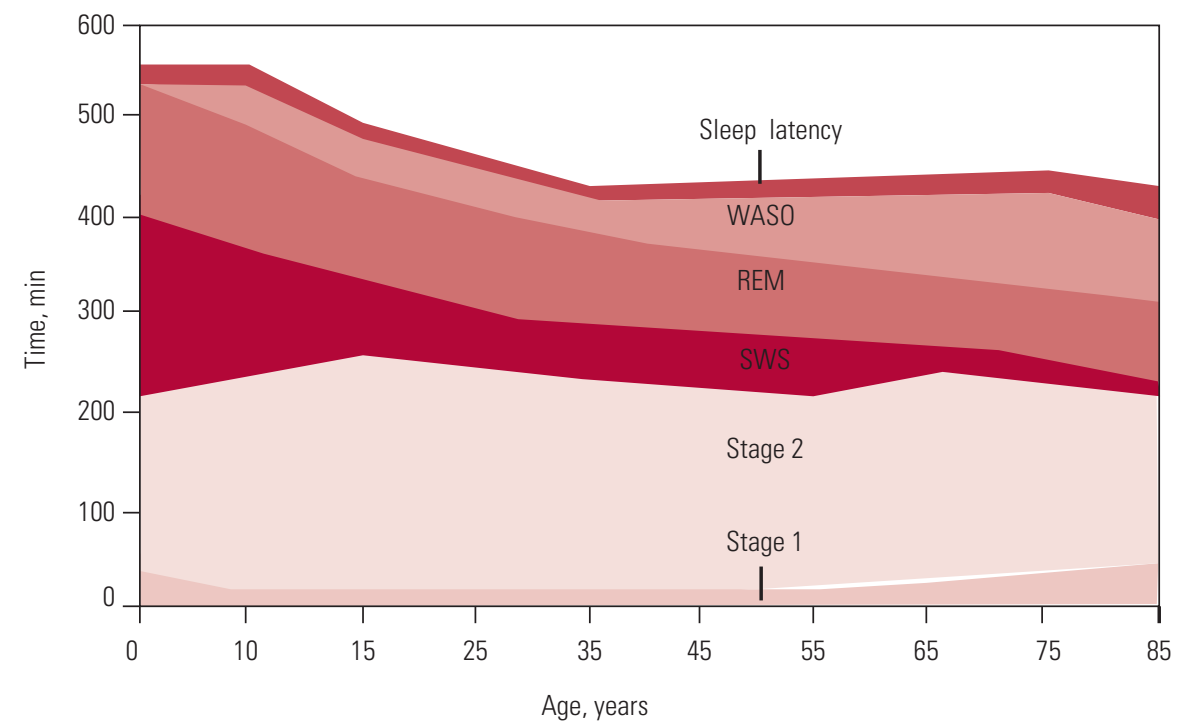

Changes in sleep with age. WASO, wake time after sleep onset; REM, rapid eye movement; SWS, slow-wave sleep (Carskadon 2005). (c) 2006 National Academy of Sciences: reproduced with permission of Elsevier. 
Circadian rhythm sleep-wake disorders are defined by ISCD-3 as 'a chronic or recurrent pattern of sleep-wake rhythm disruption primarily caused by an alteration in the circadian timing system or misalignment between the circadian rhythm and the sleep-wake schedule desired or required causing a sleep-wake disturbance (ie, insomnia or excessive sleepiness, and associated distress or impairment)' (American Academy of Sleep Medicine 2014). For all circadian rhythm sleep-wake disorders, with the exception of jet lag disorder, a duration criterion of at least 3 months is specified by ISCD-3.

\section{Sleep and its relationship to psychiatric disorders}

It is estimated that $40 \%$ of people diagnosed with insomnia have a coexisting psychiatric condition (Anderson 2013). A common symptom in the ICD10 diagnosis of a depressive episode is disturbed sleep, and epidemiological studies have found a 50-90\% comorbidity between depression and poor sleep quality (Anderson 2013). Additionally, those who have obstructive sleep apnoea are likely to experience depressive symptoms (BaHammam 2016). ICD-10 guidelines indicate that when psychiatric disorders co-occur with insomnia, both diagnoses should be given (World Health Organization 1992). In the treatment of depression, if there is a comorbid sleep disorder, the greatest risk factor for relapse is the presence of an ongoing untreated sleep disorder (Fang 2018). Other common psychiatric disorders co-occurring with sleep disorders include schizophrenia (30-80\%) and bipolar disorder (17-57\%) (Anderson 2013).

Sleep disorders are common in children with Tourette syndrome or chronic tic disorder, obsessive compulsions, anxiety, ADHD and ASD. A systematic review reported that sleep difficulties occur in 9.7$80.4 \%$ of children with Tourette syndrome/chronic tic disorder, with co-occurring anxiety increasing the risk of sleep disorders (Hibberd 2020).

Early evidence suggests that mental disorders improve when interventions for sleep disorders are provided. The SENSE study was a randomised controlled trial including 144 adolescents (12-17 years) with high levels of anxiety and sleeping difficulties without a past or current depressive disorder (Blake 2016). Following a cognitive-behavioural/ mindfulness-based intervention for sleep disturbance, improvements were noted on subjective and objective sleep measures and in anxiety symptoms (Blake 2016). OASIS, a randomised controlled trial of cognitive-behavioural therapy (CBT) for insomnia involving 3755 university students resulted in a reduction in paranoia and hallucinatory experiences (Freeman 2017).

\section{Autism spectrum disorder}

Autism spectrum disorder (ASD) is a behaviourally diagnosed neurodevelopmental disorder with qualitative differences in three domains. The domains remain similar across DSM-5 (American Psychiatric Association 2013) and ICD-10 (World Health Organization 1992), and they include reciprocal social interaction, social communication and restricted, repetitive behaviour or interests. Despite the use of differing language across ICD-10 and DSM-5, the core characteristics remain the same. Severity of symptoms, comorbid intellectual impairment and daily functioning often dictate the terms used to describe ASD. Currently, childhood autism, atypical autism and Asperger syndrome exist in ICD-10, but the use of these terms is starting to reduce in clinical practice. Current clinical practice reflects DSM-5 diagnosing of all of these presentations as 'autism spectrum disorder'. The estimated prevalence of ASD in children is 2.24-3.2\%, with an increasing trend in cumulative incidence since 1992 (Zablotsky 2015; Saito 2020).

\section{Diagnosis of sleep disorders in people with ASD}

The prevalence of sleep disorders in ASD is 50 $80 \%$ in adults and $40-80 \%$ in children (Charrier 2017; Papadopoulos 2019). There are various hypotheses regarding intrinsic and extrinsic causes for the high prevalence of insomnia in individuals with ASD (Souders 2017). These include neurobiological, medical, behavioural and cultural mechanisms, and the cause in any given individual is likely to be multifactorial (Souders 2017). The prevalence of sleep disorders in ASD may be misleading owing to the lack of consensus regarding their definition (Richdale 2009). The need to assess sleep in those with ASD is highlighted in primary care prescribing. A study of prescribing practices in the UK found that the most commonly prescribed medication (including melatonin) for individuals with ASD is for sleep, and even then rates were low (9.7\% of the cohort) (Murray 2014). Insomnia is particularly prevalent in children with ASD, resulting in increasing severity of ASD symptoms (Veatch 2017). Children's sleep difficulties can have an impact on the family, affecting parents' sleep and mood and resulting in fatigue and strain on relationships. A comparison of sleep patterns between parents of neurotypical children and those with ASD identified reduced sleep quality and quantity in the latter group (Meltzer 2008).

It has been suggested that, for some children with ASD, sleep disorders occur as a result of inappropriate sleep-related behaviours and parental mismanagement (Wiggs 2004). In adults with ASD, 
objective evaluation of sleep measures indicates longer sleep onset latency and shorter sleep duration compared with neurotypical controls. Furthermore, some circadian rhythm sleep-wake disorders are significantly more likely in people with ASD than in the general population (Baker 2017). Finally. it is worth noting that parasomnias are more common in children with ASD (Ming 2009).

Diagnosis of the various sleep disorders in individuals with ASD can be difficult for a number of reasons. First, given that ASD is a social communication disorder, those who experience sleep disorders, irrespective of intellectual impairment, do not often spontaneously report sleep as a problem (Limoges 2013). Despite this, actigraphy data and subjective reporting (when explicitly asked) indicate that those with ASD experience lower rates of sleep efficiency and increased duration of sleep onset latency (Morgan 2020). Second, currently there are no validated screening tools specific to the sleep disorders experienced by those with ASD. A caution for the use of screening tools in specialist populations is that they may be misleading because they do not accurately capturing the appropriate sleep disorders relevant to that particular patient group (Faulkner 2019). Clinicians may need to take this into consideration when solely relying on screening tools for assessment or onward referral.

\section{Relationship between ASD, sleep and psychiatric disorders}

The lifetime prevalence estimates for an anxiety disorder or a depressive disorder in people with ASD are $27-43 \%$ and $23-37 \%$ respectively, which are higher than the general population (Hollocks 2019). Evidence indicates that, when sleep is improved in ASD, there are reported improvements in anxiety and depression (Loring 2018). Rates of obsessive-compulsive disorder in individuals with ASD were also significantly higher than in the general population, at 22-24\% (Hollocks 2019).

\section{Attention-deficit hyperactivity disorder}

Attention deficit hyperactivity disorder (ADHD) is characterised by the core symptoms of inattention, hyperactivity and impulsivity. There are two main sets of diagnostic criteria in current use: ICD-10 and DSM-5. Both ICD-10 and DSM-5 require 6-month duration of symptoms (National Institute for Health and Care Excellence 2018). The diagnostic criteria for ADHD have been updated in DSM-5 to capture more accurately the experience of adults affected by the disorder. DSM- 5 states that this revision is based on nearly two decades of research showing that $\mathrm{ADHD}$, although a disorder that begins in childhood, can continue through adulthood for some people (American Psychiatric Association 2013).

There is no separate diagnostic criteria for adults with ADHD in ICD-10. A diagnosis of hyperkinetic disorder may also be made in adult life using the same criteria as in children, although attention and activity must be judged with reference to developmentally appropriate norms (World Health Organization 1992).

\section{Diagnosis of sleep disorders in people with ADHD}

Sleep problems are reported in an estimated 25-50\% of individuals who have ADHD (Corkum 1998). A systematic review of sleep disorders in adolescents with ADHD identified overall association between sleep disorders and poorer outcomes in clinical, cognitive and functional domains (Lunsford-Avery 2016). Comorbidities in ADHD such as circadian rhythm sleep-wake disorders, sleep-related breathing disorders, narcolepsy and insomnia are often missed and left untreated. A review by Konofal et al (2010) reported that up to $44 \%$ of those with $\mathrm{ADHD}$ had restless legs syndrome, but the authors advised caution in interpreting this finding owing to the methodological limitations identified in the studies. However, this may be relevant from a clinical evaluation point of view. More studies are needed to understand the prevalence and underlying pathways of symptoms of restless legs syndrome in those with ADHD.

Poor sleep hygiene, disrupted routine and impulse control difficulties may affect the ability of an individual with $\mathrm{ADHD}$ to have a winding-down period, leading to bedtime resistance and delayed sleep onset. Delayed sleep-wake phase disorder and late chronotype are frequently comorbid with $\mathrm{ADHD}$ in both adults and adolescents (Kooij 2013; Sivertsen 2014). There are proposals suggesting that people with $\mathrm{ADHD}$ may have stronger delayed circadian preference and a possible delayed release of endogenous melatonin (Gruber 2012).

In $\mathrm{ADHD}$, insomnia is reported to be more frequent among children (73.3\%) (Sung 2008) than among adults (66.8\%) (Brevik 2017). These rates are higher than insomnia estimates in children (20-30\%) (Calhoun 2014) and adults (6-50\%) (Ohayon 2002) in the general population. Women with $\mathrm{ADHD}$ reported higher prevalence of insomnia than women without ADHD (43.9 v. 12.2\%) (Fuller-Thomson 2016). Recent studies indicate that over $30 \%$ of individuals with narcolepsy, which is characterised by excessive daytime sleepiness and cataplexy, have comorbid ADHD (Kim 2020). A history of snoring or possible obstructive sleep apnoea during childhood is associated with a 


\begin{tabular}{|c|c|c|}
\hline Sleep history & Nature of the sleep disturbance & Additional factors to consider \\
\hline $\begin{array}{l}\text { - Onset of the problem (acute or chronic) } \\
\text { - Duration of the problem } \\
\text { - Severity (subjective and objective) } \\
\text { - Is the problem constant or episodic? } \\
\text { - Impact on behaviour, mood, cognitive } \\
\text { functioning, academic progression } \\
\text { - Developmental appropriateness of the } \\
\text { - } \text { problem } \\
\text { - } \text { - Famontal illness) } \\
\text { - Is it iatrogenic? (medications that } \\
\text { - } \text { cause sleep impairments) } \\
\text { - Family/carer expectations }\end{array}$ & $\begin{array}{l}\text { - Sleep onset latency (difficulty falling } \\
\text { - } \text { Sleep) } \\
\text { - } \text { Sleeping) } \\
\text { - } \text { Number and duration of night-time } \\
\text { - } \text { awakenings } \\
\text { - } \text { Morning wake time } \\
\text { - } \text { Daytime napping in sleep time } \\
\text { - } \text { Restlessness, particularly legs } \\
\text { - Nightmares } \\
\text { - Unusual or bizarre behaviour at night } \\
\text { - } \text { (include night terrors and sleep paralysis) } \\
\text { Breathing difficulties or snoring (noted by } \\
\text { partner or carers) }\end{array}$ & $\begin{array}{l}\text { - Diet and timing of food (include caffeine, } \\
\text { - Allergies) } \\
\text { - Alcohol and illicit substance intake } \\
\text { - } \text { Travel, jet lag and shift working } \\
\text { telephone use, playing in bed, stereotypy) } \\
\text { - Sleeping environment (other people, noise, } \\
\text { lights, temperature, bed comfort, odour, } \\
\text { humidity) } \\
\text { - Anxiety and current stressors } \\
\text { - What has the person tried or is currently } \\
\text { trying to resolve the problem? }\end{array}$ \\
\hline
\end{tabular}

twofold difference in the odds of ADHD diagnosis or symptoms (Constantin 2014). Other psychopathologies, such as anxiety, depression and tic disorders, are common in those with $\mathrm{ADHD}$, and we recommend that a systematic assessment of coexisting conditions be carried out when evaluating for sleep disorders in those with ADHD.

\section{The relationship between $A D H D$ and $A S D$}

Considerable overlap exists between ASD and other mental disorders. ADHD, anxiety disorders, depression, conduct disorder and behaviours that challenge are among the disorders most commonly associated with ASD (Matson 2013). DSM-5 permits a dual diagnosis of both ADHD and ASD. An estimated $30-80 \%$ of children with ASD also meet the criteria for $\mathrm{ADHD}$ and, conversely, $20-50 \%$ of children with ADHD meet criteria for ASD. Given this overlap, more research is needed to further understand the relationship between the two conditions. It is important that clinicians are aware of the conditions likely to co-occur with ASD, which vary according to the person's age. Clinicians should also be familiar with the assessment methods that are available to assist in diagnosis and keep up to date with new research in this area (Matson 2013).

\section{Assessment and diagnosis}

Before assessing for a sleep disorder, the clinician should consider predisposing, precipitating and perpetuating factors that cause sleep disturbance. An example is gastrointestinal disorders, which are commonly associated with sleep problems in individuals with ASD (Klukowski 2015). Additionally, behaviours that challenge may need considering as a cause of the sleep disorder and can be measured using an appropriate screening tool. The first step in assessing a sleep disorder in children and adults with $\mathrm{ASD}$ and $\mathrm{ADHD}$ is taking a detailed sleep history. There are a range of factors to consider, outlined in Table 1.

Taking a detailed history may highlight factors that clearly indicate environmental or iatrogenic impact on sleep. The flowchart shown in Fig. 3 can be followed to inform the decision to diagnose or move to a specialist referral.

\section{Screening tools}

Box 1 lists frequently used questionnaires for screening for clinically significant sleep problems. These tools can be found online and some are available to use for clinical and research purposes free of charge.

\section{Specialist sleep services}

In some cases, it may be necessary to refer individuals with ASD or ADHD for assessment at a specialist centre. Sleep centres in the UK can be located through the National Health Service's 'Find Sleep Medicine Services' webpage (www.nhs. uk/service-search/other-services/Sleep-Medicine/ LocationSearch/1888). As a rule of thumb, any new onset of parasomnia should trigger further evaluation by a specialist. Table 2 provides an overview of the signs and symptoms that indicate that referral to a specialist centre may be necessary.

Diagnosis of a sleep disorder Typically, specialist services will identify and diagnose a sleep disorder by taking a detailed history (especially about the sleep problem), performing a physical and behavioural examination, and monitoring the $24 \mathrm{~h}$ sleep-wake pattern using a sleep diary and possibly objective sleep studies such as actigraphy or polysomnography (Stores 2015). Information about family circumstances and history will also be 


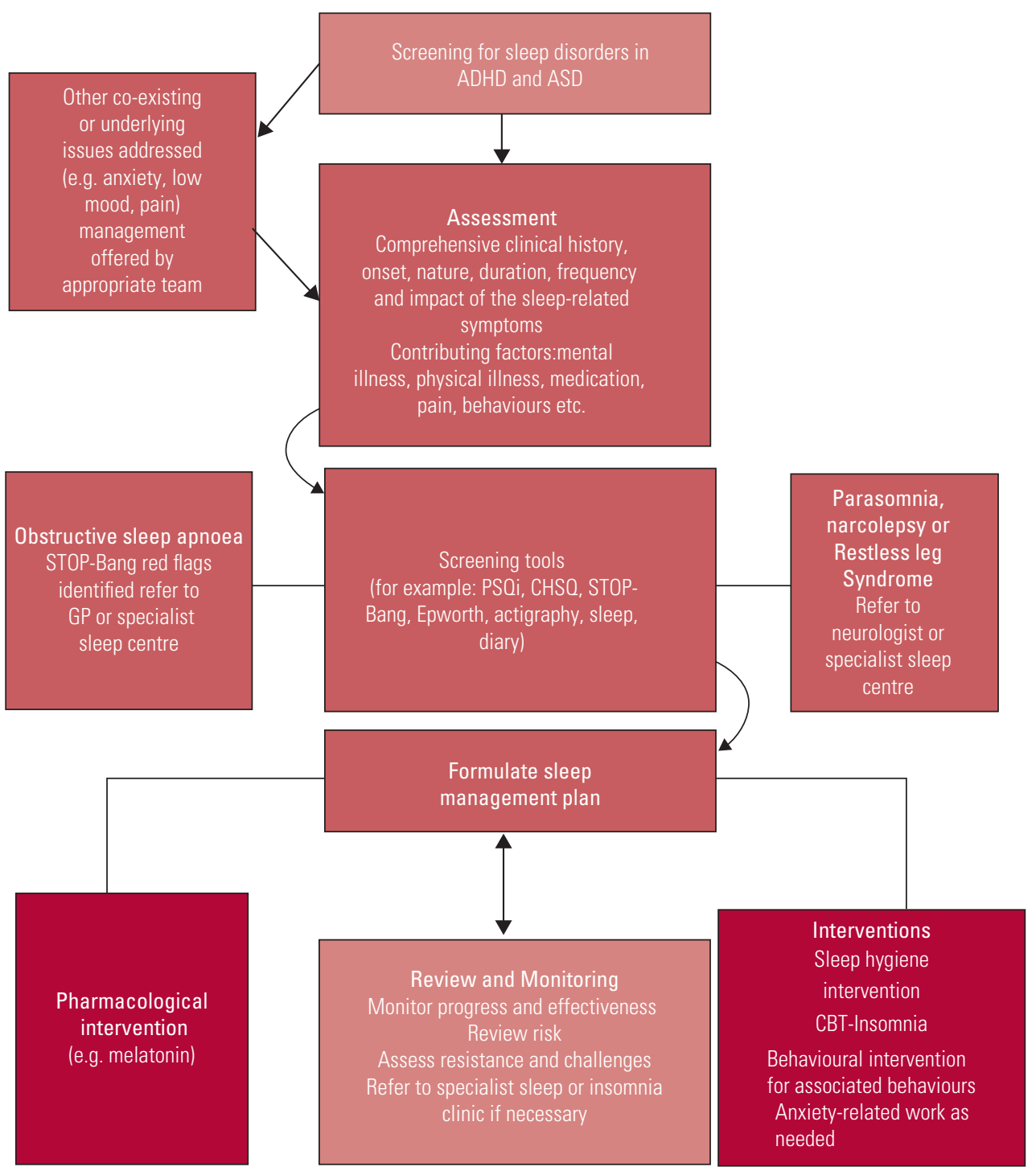

Flowchart for the assessment of sleep disorders. ADHD, attention-deficit hyperactivity disorder; ASD, autism spectrum disorder; GP, general practitioner; PSQi, Pittsburgh Sleep Quality Index; CHSQ, Children's Sleep Habits Questionnaire; $\mathrm{CBT}$, cognitive-behavioural therapy.

sought. For children, parenting practices and the child's development history will be assessed. Figure 4 outlines patterns observed in common circadian rhythm sleep-wake disorders.

\section{Treatment options}

One of the problems noted in the literature is the use of an array of terminologies to describe sleep symptoms, such as sleep disturbance, sleep difficulties and sleep disorders. Making a clear distinction between them will be useful in clinical practice. Aspects of sleep should be carefully assessed before starting treatment or medication. This requires the clinician to use structured assessments and evaluation tools, which will help them to understand the baseline state and choose a suitable treatment option.

\section{Non-pharmacological interventions}

In the management of insomnia and circadian rhythm sleep-wake disorders in individuals with $\mathrm{ADHD}$ or ASD, lifestyle factors, including exercise and diet, should be considered, followed by consideration of strategies to ensure adherence to treatment plans (National Institute for Health and Care Excellence 2018). The use of personalised reminders, incentives and clearly documented instructions may help (Hauck 2016). Social Stories ${ }^{\mathrm{TM}}$ (Kokina 2010) and visual schedules (Malow 2014) have shown good short-term effectiveness in reducing behaviours that challenge related to bedtime routines and facilitating interventions such as the bedtime pass programme for children.

Interventions for children with ASD primarily focus on education of parents and carers (Johnson 
BOX 1 Commonly used screening questionnaires for clinically significant sleep problems

For children and adolescents

- Bespoke sleep diary (1 week minimum)

- Sleep Disturbance Index (SDI): a four-item, parent-rated questionnaire that covers settling problems, night waking and parental involvement at night.

- Children's Sleep Habits Questionnaire (CSHQ): a 45-item, parent-rated questionnaire that assesses the frequency of behaviours associated with common paediatric sleep disorders.

- Paediatric Daytime Sleepiness Scale (PDSS): self-report questionnaire designed to assess sleepiness in 11- to 15year-olds.

For adults
- Epworth Sleepiness Scale (ESS): a self-administered questionnaire with eight questions. Respondents are asked to rate, on a four-point scale (0-3), their usual chances of dozing off or falling asleep while engaged in eight different activities.

- Pittsburgh Sleep Quality Index (PSQI): a self-report questionnaire that assesses sleep quality over a 1-month period.

- STOP-Bang Questionnaire: an eight-item questionnaire that determines risk for sleep apnoea. It is one of the most widely accepted screening tools for obstructive sleep apnoea.

- Morningness-Eveningness Questionnaire (MEQ): a selfassessment questionnaire to measure whether a person's circadian rhythm (biological clock) produces peak alertness in the morning, in the evening or in between.

- Bespoke sleep diary (1 week minimum)

2013). Training programmes include providing information about normal sleep, recommendations for establishing good stimulus control in the bedroom environment, sleep scheduling and managing maladaptive behaviour at night-time (Johnson 2013). Providing two educational sessions covering sleep hygiene and relaxation strategies to adolescents with ASD and their carers resulted in improvements in sleep latency and efficiency (Loring 2018). In all interventions outlined above, homework tasks are set and reviewed at subsequent sessions (Cortesi 2012; Loring 2018). Online parent training has also shown promising short-term sleep improvements in children with ASD (Roberts 2019).

Sleep hygiene is a term used widely in sleep disorders, but it will look different for each patient. It focuses on the principles of stimulus control and nurturing bodily changes to encourage sleep during natural cycles. Stimulus control refers to ensuring that the place of sleep is associated with sleeping and other places are associated with wakefulness. An example of an intervention to promote stimulus control among adolescents or adults is leaving the bedroom after $15 \mathrm{~min}$ if not sleeping and then returning to bed only when tired. Any non-sleep-related behaviours that occur in bed (e.g. laptop use, video games or watching TV) should be relocated to other living areas where possible.

In adults with ASD and ADHD, as circadian rhythm sleep-wake disorders are more common, the use of light therapy has shown effectiveness, involving 10000 lux light to promote wakefulness and 400 lux dim red light prior to sleep onset. In advanced sleep-wake phase disorder, 10000 lux can be considered between 18.00 and $21.00 \mathrm{~h}$; for delayed sleep-wake phase disorder 10000 lux can be considered between 10.00 and $13.00 \mathrm{~h}$; and for irregular sleep-wake disorder 10000 lux at $09.00 \mathrm{~h}$ for 30 minutes might be used (Burgess 2016). There is growing evidence for the use of CBT for insomnia (CBT-I) face to face or online (National Institute for Health and Care Excellence 2017). A consideration that might affect the likelihood of behaviour change in individuals with ASD or $\mathrm{ADHD}$ is the extent to which restricted and repetitive behaviours affect daily living and sleep routines.

Finally, sleep disorders are highly associated with daytime distress and maladaptive behaviours in individuals with ASD (Abel 2018). In adults as well as children with a diagnosis of ASD, a functional analysis of behaviours that challenge followed by a function-based intervention plan is recommended (National Institute for Health and Care Excellence 2012). If a functional analysis is not feasible, other functional assessment procedures can be used aiming to understand the relationship between the maladaptive behaviour and its maintaining consequences. In adults, who are very likely to be able to describe the contingencies, this may be sufficient to understand maintaining consequences.

A recent systematic review of non-pharmacological interventions for non-respiratory sleep disorders in children with neurodisabilities (including ASD and ADHD) identified that, despite clinical guidance recommending parent-directed interventions as the first approach to managing sleep disorders, there is a lack of high-quality evidence assessing the effectiveness of non-pharmacological interventions for these populations (Scantlebury 2018). Given the limitations of the existing evidence, 


\begin{tabular}{|c|c|c|}
\hline $\begin{array}{l}\text { Sleep disturbance/ } \\
\text { disorder }\end{array}$ & Description & $\begin{array}{l}\text { Signs and symptoms that indicate referral to a } \\
\text { specialist centre may be necessary }\end{array}$ \\
\hline Nightmares & $\begin{array}{l}\text { Repeated occurrences of extended, extremely } \\
\text { dysphoric and well-remembered dreams that } \\
\text { usually involve threats to survival, security or } \\
\text { physical integrity }\end{array}$ & $\begin{array}{l}\text { May require further investigation if there are } \\
\text { unusual features such as drooling, stiffening, } \\
\text { jerking, repetitive screaming, kicking or } \\
\text { thrashing. Referral may also be necessary if } \\
\text { there is a history of significant head injury }\end{array}$ \\
\hline $\begin{array}{l}\text { Sleep-walking or night } \\
\text { terrors }\end{array}$ & $\begin{array}{l}\text { Undesirable physical events or experiences that } \\
\text { occur during the initiation of sleep, during sleep } \\
\text { or during arousal from sleep }\end{array}$ & $\begin{array}{l}\text { May require further investigation if the onset is } \\
\text { during adolescence, as this may indicate an } \\
\text { underlying medical or psychological problem. } \\
\text { Also refer if the individual is a danger to } \\
\text { themselves or others or if the episodes cause } \\
\text { significant disruption or distress }\end{array}$ \\
\hline Sleep apnoea & $\begin{array}{l}\text { Temporary cessation of breathing during sleep. } \\
\text { Sleep-related breathing disorders are divided } \\
\text { into four sections: obstructive sleep apnoea, } \\
\text { central sleep apnoea syndromes, sleep-related } \\
\text { hypoventilation disorders, and sleep-related } \\
\text { hypoxaemia disorder }\end{array}$ & $\begin{array}{l}\text { Consider referral if there are concerns about } \\
\text { breathing difficulties, excessive daytime } \\
\text { tiredness or sleep disturbance with snoring. } \\
\text { Refer or liaise with general practitioner if there } \\
\text { are red flags on the STOP-Bang questionnaire. } \\
\text { Also refer if blue lips (cyanosis) have been } \\
\text { reported by parents or carers }\end{array}$ \\
\hline Bruxism & $\begin{array}{l}\text { Involuntary habitual grinding of the teeth, typically } \\
\text { during sleep }\end{array}$ & $\begin{array}{l}\text { Refer if there are concerns about underlying stress } \\
\text { or a dental problem }\end{array}$ \\
\hline Narcolepsy & $\begin{array}{l}\text { Chronic neurological sleep disorder characterised by } \\
\text { excessive daytime sleepiness, cataplexy (loss of } \\
\text { muscle tone) and disturbed night-time sleep }\end{array}$ & $\begin{array}{l}\text { Refer if the following features are reported: } \\
\text { excessive daytime sleepiness (EDS), which can } \\
\text { be assessed using the Epworth Sleepiness } \\
\text { Scale (score of }>10 \text { indicates EDS and }>17 \\
\text { indicates severe EDS), cataplexy features, } \\
\text { hallucinations, weight gain, sleep paralysis }\end{array}$ \\
\hline Movement disorders & $\begin{array}{l}\text { Leg movement disorders and rhythmic movement } \\
\text { disorders (as listed below) }\end{array}$ & $\begin{array}{l}\text { Refer if causing disturbance to sleep, family life or if } \\
\text { there is a suggestion of an underlying medical } \\
\text { trigger }\end{array}$ \\
\hline Restless legs syndrome & $\begin{array}{l}\text { Also known as Willis-Ekbom disease: a common } \\
\text { condition of the nervous system that causes an } \\
\text { overwhelming irresistible urge to move the legs }\end{array}$ & $\begin{array}{l}\text { Refer if causing significant pain, sleep disruption or } \\
\text { distress }\end{array}$ \\
\hline $\begin{array}{l}\text { Periodic limb movement } \\
\text { syndrome }\end{array}$ & $\begin{array}{l}\text { Repetitive cramping or jerking of the legs during } \\
\text { sleep }\end{array}$ & Refer if causing significant disturbance to sleep \\
\hline $\begin{array}{l}\text { Rhythmic movement } \\
\text { disorders } \\
\text { (headbanging, body } \\
\text { rocking) }\end{array}$ & $\begin{array}{l}\text { Sleep-related rhythmic movement disorder involving } \\
\text { repeated body movements }\end{array}$ & $\begin{array}{l}\text { More common in autism spectrum disorder. Refer if } \\
\text { there are concerns about possible abuse, as } \\
\text { this can be related to physical, sexual or } \\
\text { emotional abuse }\end{array}$ \\
\hline $\begin{array}{l}\text { Rapid eye movement } \\
\text { (REM) sleep } \\
\text { behaviour disorder }\end{array}$ & $\begin{array}{l}\text { Dream re-enactment behaviour during REM sleep } \\
\text { that can result in injury to self and others }\end{array}$ & $\begin{array}{l}\text { Refer to a specialist sleep clinic. Also, consider } \\
\text { iatrogenic effects of tricyclic antidepressants, } \\
\text { monoamine oxidase inhibitors and selective } \\
\text { serotonin reuptake inhibitors }\end{array}$ \\
\hline
\end{tabular}

the authors highlighted the importance of prioritising research in this area.

\section{Pharmacological interventions}

From a clinical perspective when prescribing, thorough consideration must be given to predisposing, precipitating and perpetuating factors and differential diagnosis. Some psychiatric drugs have insomnia as a side-effect or even unmask a subclinical REM sleep behaviour disorder. If there are underlying coexisting mental illnesses such as anxiety or depression, the choice of medication with sedative effects may be worth considering, but not as a stand-alone treatment for sleep disorders.

Stimulants are the first-line medication for treatment of ADHD. The effects of stimulant medication on sleep in those with ADHD vary, which may be a reflection of the underlying complexity of the link between $\mathrm{ADHD}$ and sleep disorders (Graham 2011). Stimulants are associated with disturbed sleep, such as increased sleep onset latency, difficulties falling asleep and decreased total sleep time in those with ADHD (Stein 2012). However, some studies have also shown that $\mathrm{ADHD}$ symptom control can promote sleep (Jerome 2001). Adjusting the dose or changing to another formulation have all been proposed (Konofal 2010). In contrast to stimulants, the most common side-effect associated with atomoxetine and guanfacine is somnolence. Dosing in the evening rather than in the morning has been found to reduce daytime somnolence with atomoxetine (Block 2009).

Medications can be effective in treating circadian rhythm sleep-wake disorders and insomnia. Melatonin has been shown to reduce the symptoms 

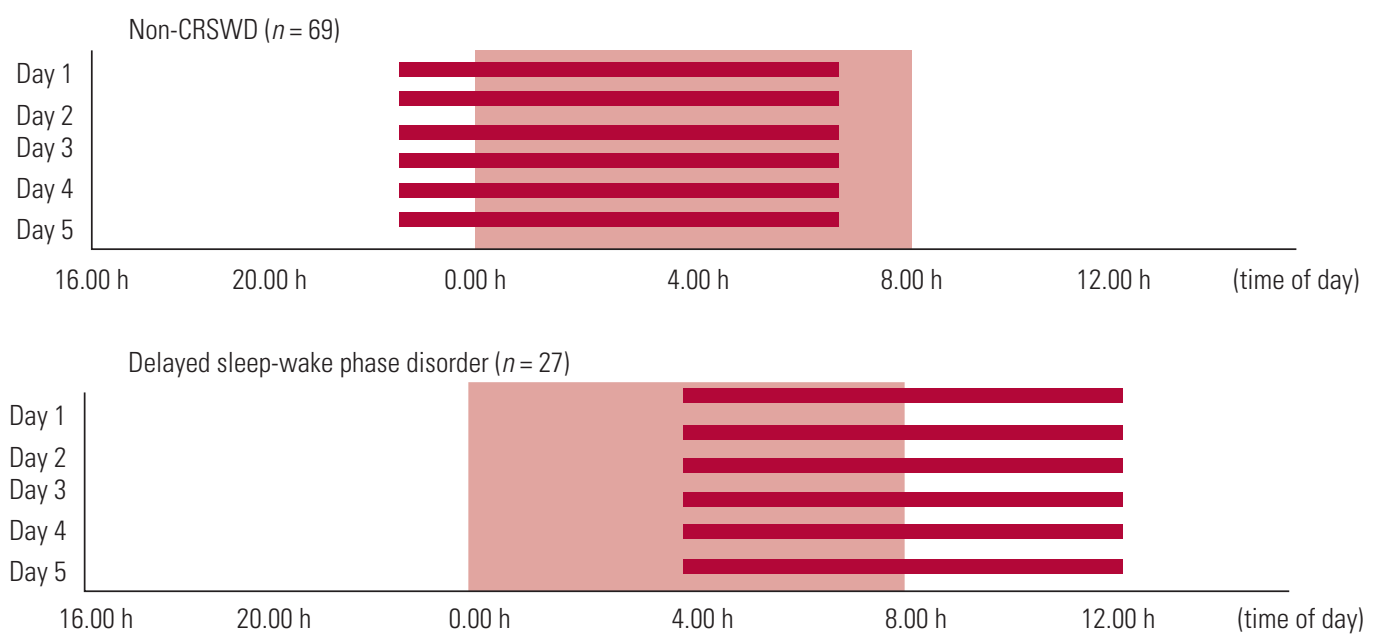

Non-24 hour sleep-wake rhythm disorder $(n=6)$

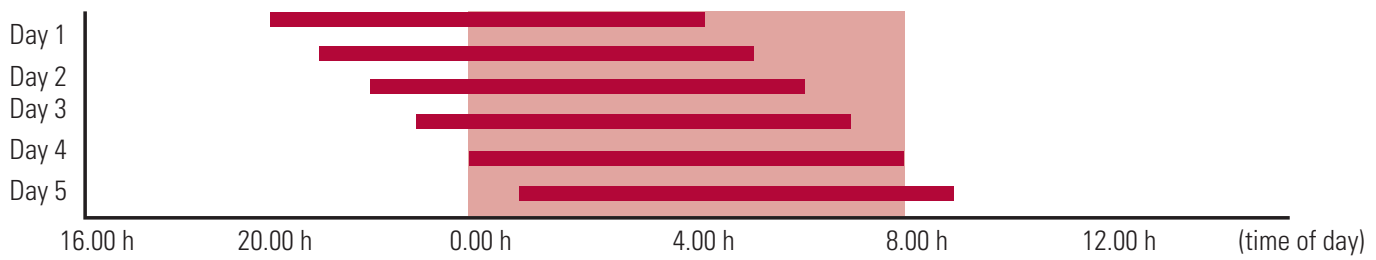

Irregular sleep-wake rhythm disorder $(n=2)$

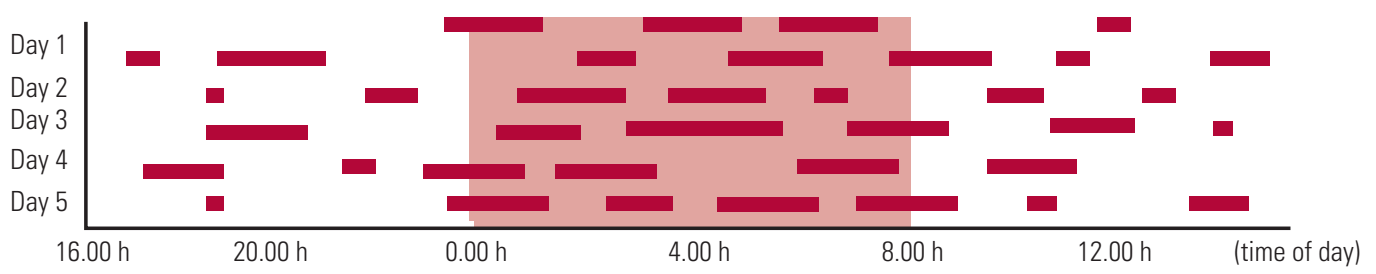

FIG 4 Circadian rhythm sleep-wake disorders (CRSWD) (Takaesu 2016). (C) 2016 Takaesu et al: reproduced under the terms of the Creative Commons Attribution License (CC BY 4.0).

related to insomnia, to improve sleep latency and improve sleep efficiency and total sleep duration (Jenabi 2019). This observation was replicated in a systematic review of pharmacological treatments for sleep disorders in children, which concluded that melatonin was useful in improving sleep latency, sleep duration and wake time after sleep onset at least in the short term, particularly with children with comorbid ASD and other neurodevelopmental disorders (McDonagh 2019). However, there was no improvement noted in the number of awakenings per night with melatonin. More research is required to establish how and when to stop melatonin use in individuals with neurodevelopmental disorders. Utilising melatonin should be considered with caution as it is not currently licensed in the UK for use in adults between 18 and 55 years of age (National Institute for Health and Care Excellence 2015).

Hypnotics are effective in treatment of insomnia, however they are for short-term use and only work for as long as they are used (Riemann 2009). Short-acting hypnotics may be helpful for sleeponset insomnia. In the UK, hypnotics for the management of insomnia are only licensed for short-term use. Clinicians should consider the risk of sideeffects, including dependency, cognitive impairment, falls and withdrawal symptoms. NICE guidance suggests only to use hypnotics if insomnia is severe and to use the lowest possible dose for the shortest period of time (National Institute for Health and Care Excellence 2015). Insomnia can be a chronic condition, so behavioural interventions and CBT-I are suggested as treatment choices (Riemann 2009).

\section{Neurodevelopmental disorders and sleep disorders in intellectual disabilities}

For individuals with intellectual disabilities, there is a fourfold higher likelihood of having ADHD than for the general population (Axmon 2018). There is 
an even greater likelihood of having a comorbid diagnosis of ASD. The prevalence of sleep disorders among people with intellectual disabilities is high and increased further in populations with specific chromosomal disorders (Boyle 2010).

Sleep disorders in this population can present as behaviours that challenge and are associated with poor quality of life for the patient and caregivers (Lenjavi 2010). Current guidance for adults with intellectual disabilities highlights the completion of a functional analysis prior to the use of melatonin (National Institute for Health and Care Excellence 2015). However, there is no evidence for the use of functional analysis in identifying or assessing sleep-related behaviours that challenge and, as mentioned above, melatonin only has a licence for those under 18 and over 55 (National Institute for Health and Care Excellence 2015). Before assessment for a sleep disorder, a comprehensive assessment of physical health, including review of current medications and epilepsy management, may be needed.

A systematic review of interventions for sleep difficulties, including sleep hygiene, behavioural interventions and use of melatonin, found that existing literature is of low quality (Shanahan 2019). However, there is some evidence that educating parents about interventions such as stimulus control and other sleep-related behaviours improves sleep in children and should be considered for parents and carers of adults with ASD and comorbid intellectual disabilities.

\section{Clinical implications and future directions}

Children and adults presenting with neurodevelopmental disorders should be offered assessment for primary sleep disorders which may need separate treatment strategies. The problems of circadian rhythm sleep-wake disorders and insomnia can persist into adulthood if not treated. The focus questions outlined in Table 1 may assist in taking the clinical history. Baseline records of sleep onset latency, night-to-night variability and total sleep duration before treatment begins will be helpful to evaluate progress and formulation of a management plan. Most intervention plans should include psychoeducation and behavioural interventions such as sleep hygiene. Sleep studies may be required for those with new or complex sleep disorders.

Clinicians may face additional challenges on continued prescriptions of melatonin. Shared-care treatment protocols and transition meetings between children's and adult services or primary care physicians may be required.

A diagnosis of chronic insomnia disorder should only be considered when the insomnia is especially prominent or unexpectedly prolonged and is the focus of clinical assessment and treatment. In such cases, care is best offered in consultation with sleep clinics.

Behavioural interventions should be considered for long-term sleep disorders. One such is CBT for insomnia (CBT-I), an evidence-based effective treatment for insomnia in those with comorbid psychiatric illness (Taylor 2014). There are several webbased apps available, such as Sleepio, Headspace and Calm, which incorporate aspects of CBT, relaxation, meditation and mindfulness. There is insufficient evidence supporting the use of apps such as Sleepio, Headspace and Calm in this population and there are risks of high drop-out rates (Freeman 2017; National Institute for Health and Care Excellence 2017). More research into the effectiveness of online CBT-I or sleep apps should be considered, given the high likelihood of cost savings and benefits of a non-face-to-face approach for people with ASD and also in situations that restrict a face-to-face consultation, such as pandemic. Longitudinal research is required to inform the adaptation of pharmacological and non-pharmacological sleep interventions to better meet the needs of children and adults with ADHD or ASD and their families (Martin 2019).

Clinical evaluations and management plans based on comprehensive assessment and multidisciplinary team evaluation, timely consultations with other appropriate specialists in primary care, neurology and respiratory medicine, and referral to specialist sleep centres remain the mainstay in identifying and managing sleep disorders in people with ASD or ADHD.

\section{Acknowledgements}

We thank Dr Swapnil Palod (Mackay Base Hospital, Queensland, Australia) for his contribution to information about prescribing and initial coordination of the project.

\section{Author contributions}

All three authors contributed to writing sections of this article.

\section{Declaration of interest}

None.

ICMJE forms are in the supplementary material, available online at https://doi.org/10.1192/bja. 2020.65 .

\section{References}

Abel EA, Schwichtenberg AJ, Brodhead MT, et al (2018) Sleep and challenging behaviors in the context of intensive behavioral intervention for children with autism. Journal of Autism and Developmental Disorders, 48: $3871-84$
MCO answers

$1 \mathrm{~d} 2$ e 3 e 4 b $5 c$ 
Altevogt BM, Colten HR (2006) Sleep Disorders and Sleep Deprivation: An Unmet Public Health Problem. National Academies Press.

American Academy of Sleep Medicine (2014) The International Classification of Sleep Disorders - Third Edition (ICSD-3). American Academy of Sleep Medicine.

American Psychiatric Association (2013) Diagnostic and Statistical Manual of Mental Disorders (5th edn) (DSM-5). American Psychiatric Publishing.

Anderson KN, Bradley AJ (2013) Sleep disturbance in mental health problems and neurodegenerative disease. Nature and Science of Sleep, 5 : $61-75$.

Axmon A, Björne P, Nylander L, et al (2018) Psychiatric diagnoses in older people with intellectual disability in comparison with the general population: a register study. Epidemiology and Psychiatric Sciences, 27: 479-91.

BaHammam AS, Kendzerska T, Gupta R, et al (2016) Comorbid depression in obstructive sleep apnea: an under-recognized association. Sleep and Breathing, 20: 447-56.

Baker EK, Richdale AL (2017) Examining the behavioural sleep-wake rhythm in adults with autism spectrum disorder and no comorbid intellectual disability. Journal of Autism and Developmental Disorders, 47: $1207-22$

Blake M, Waloszek JM, Schwartz 0, et al (2016) The SENSE study: post intervention effects of a randomized controlled trial of a cognitive-behav ioral and mindfulness-based group sleep improvement intervention among at-risk adolescents. Journal of Consulting and Clinical Psychology, 84: 1039-51

Block SL, Kelsey D, Coury D, et al (2009) Once-daily atomoxetine for treating pediatric attention-deficit/hyperactivity disorder: comparison of morning and evening dosing. Clinical Pediatrics, 48: 723-33.

Boyle A, Melville C, Morrison J, et al (2010) A cohort study of the prevalence of sleep problems in adults with intellectual disabilities. Journal of Sleep Research, 19: 42-53.

Brevik E, Lundervold A, Halmøy A, et al (2017) Prevalence and clinical correlates of insomnia in adults with attention-deficit hyperactivity disorder. Acta Psychiatrica Scandinavica, 136: 220-7.

Burgess HJ, Emens JS (2016) Circadian-based therapies for circadian rhythm sleep-wake disorders. Current Sleep Medicine Reports, 2: 158-65.

Calhoun S, Fernandez-Mendoza J, Vgontzas A, et al (2014) Prevalence of insomnia symptoms in a general population sample of young children and preadolescents: gender effects. Sleep Medicine, 15: 91-5.

Cappuccio FP, D'Elia L, Strazzullo P, et al (2010) Sleep duration and allcause mortality: a systematic review and meta-analysis of prospective studies. Sleep, 33: 585-92.

Cappuccio FP, Cooper D, Delia L, et al (2011) Sleep duration predicts cardiovascular outcomes: a systematic review and meta-analysis of prospective studies. European Heart Journal, 32: 1484-92.

Carskadon M, Dement W (2005) Normal human sleep: An overview. In Principles and Practice of Sleep Medicine 4th ed (eds MH Kryger, T Roth, WC Dement): 13-23. Elsevier Saunders

Charrier A, Olliac B, Roubertoux P, et al (2017) Clock genes and altered sleep-wake rhythms: their role in the development of psychiatric disorders. International Journal of Molecular Sciences, 18: 938.

Constantin E, Low N, Dugas E, et al (2014) Association between childhood sleep-disordered breathing and disruptive behavior disorders in childhood and adolescence. Behavioral Sleep Medicine, 13: 442-54.

Corkum P, Tannock R, Moldofsky H (1998) Sleep disturbances in children with attention-deficit/hyperactivity disorder. Journal of the American Academy of Child \& Adolescent Psychiatry, 37: 637-46.

Cortesi F, Giannotti F, Sebastiani T, et al (2012) Controlled-release melatonin, singly and combined with cognitive behavioural therapy, for persistent insomnia in children with autism spectrum disorders: a randomized placebo-controlled trial. Journal of Sleep Research, 21: 700-9.

Dunlap JC, Loros JJ, DeCoursey PJ (2004) Chronobiology: Biological Timekeeping. Sinauer Associates.

Fang H, Tu S, Sheng J, et al (2018) Depression in sleep disturbance: a review on a bidirectional relationship, mechanisms and treatment. Journal of Cellular and Molecular Medicine, 23: 2324-32.
Faulkner SM, Sidey-Gibbons C (2019) Use of the Pittsburgh Sleep Quality Index (PSOI) in people with schizophrenia spectrum disorders: a mixed methods study. Frontiers in Psychiatry, 10: 284.

Freeman D, Sheaves B, Goodwin GM, et al (2017) The effects of improving sleep on mental health (OASIS): a randomised controlled trial with mediation analysis. Lancet Psychiatry, 4: 749-58.

Fuller-Thomson E, Lewis D, Agbeyaka S (2016) Attention-deficit/hyperactivity disorder casts a long shadow: findings from a population-based study of adult women with self-reported ADHD. Child: Care, Health and Development, 42: 918-27.

Graham J, Banaschewski T, Buitelaar J, et al (2011) European guidelines on managing adverse effects of medication for ADHD. European Child \& Adolescent Psychiatry, 20: 17-37.

Gruber R, Fontil L, Bergmame $L$ (2012) Contributions of circadian tendencies and behavioral problems to sleep onset problems of children with ADHD. BMC Psychiatry, 12: 212

Hauck JL, Ketcheson LR, Ulrich DA (2016) Methodology to promote physical activity monitoring adherence in youth with autism spectrum disorder. Frontiers in Public Health, 4: 206.

Hibberd C, Charman T, Bhatoa RS, et al (2020) Sleep difficulties in children with Tourette syndrome and chronic tic disorders: a systematic review of characteristics and associated factors. Sleep, 43(6): zsz308.

Hollocks MJ, Lerh JW, Magiati I, et al (2019) Anxiety and depression in adults with autism spectrum disorder: a systematic review and meta-analysis. Psychological Medicine, 49: 559-72

Irwin MR, Olmstead R, Carroll JE (2016) Sleep disturbance, sleep duration, and inflammation: a systematic review and meta-analysis of cohort studies and experimental sleep deprivation. Biological Psychiatry, 80: $40-52$

Jenabi E, Ataei S, Bashirian S (2019) Evaluation of drug interventions for the treatment of sleep disorders in children with autism spectrum disorders: a systematic review. Korean Journal of Pediatrics, 62: $405-9$

Jerome $L$ (2001) Can methylphenidate facilitate sleep in children with attention deficit hyperactivity disorder? Journal of Child and Adolescent Psychopharmacology, 11: 109-9.

Johnson CR, Turner KS, Foldes E, et al (2013) Behavioral parent training to address sleep disturbances in young children with autism spectrum disorder: a pilot trial. Sleep Medicine, 14: 995-1004.

Kim J, Lee GH, Sung SM (2020) Prevalence of attention deficit hyperactivity disorder symptoms in narcolepsy: a systematic review. Sleep Medicine, 65: 84-8.

Klukowski M, Wasilewska J, Lebensztejn D (2015) Sleep and gastrointestinal disturbances in autism spectrum disorder in children. Developmental Period Medicine, 19: 157-61.

Kokina A, Kern L (2010) Social Story ${ }^{\mathrm{TM}}$ interventions for students with autism spectrum disorders: a meta-analysis. Journal of Autism and Developmental Disorders, 40: 812-26.

Konofal E, Lecendreux M, Cortese S (2010) Sleep and ADHD. Sleep Medicine, 11: 652-8.

Kooij J, Bijlenga D (2013) The circadian rhythm in adult attention-deficit/ hyperactivity disorder: current state of affairs. Expert Review of Neurotherapeutics, 13: 1107-16.

Lenjavi MR, Ahuja MA, Touchette PE, et al (2010) Maladaptive behaviors are linked with inefficient sleep in individuals with developmental disabilities. Journal of Neurodevelopmental Disorders, 2: 174-80.

Limoges E, Bolduc C, Berthiaume C, et al (2013) Relationship between poor sleep and daytime cognitive performance in young adults with autism. Research in Developmental Disabilities, 34: 1322-35

Loring WA, Johnston RL, Shui AM, et al (2018) Impact of a brief behavioral intervention for insomnia on daytime behaviors in adolescents with autism spectrum disorders. Journal of Contemporary Psychotherapy, 48: 165-77.

Lunsford-Avery J, Krystal A, Kollins S (2016) Sleep disturbances in adolescents with ADHD: a systematic review and framework for future research. Clinical Psychology Review, 50: 159-74. 
Malow BA, Adkins KW, Reynolds A, et al (2014) Parent-based sleep education for children with autism spectrum disorders. Journal of Autism and Developmental Disorders, 44: 216-28.

Martin CA, Papadopoulos N, Chellew T, et al (2019) Associations between parenting stress, parent mental health and child sleep problems for children with ADHD and ASD: systematic review. Research in Developmental Disabilities, 93: 103463.

Matson JL, Williams LW (2013) Differential diagnosis and comorbidity: distinguishing autism from other mental health issues. Neuropsychiatry, 3: 233-243.

McDonagh MS, Holmes R, Hsu F (2019) Pharmacologic treatments for sleep disorders in children: a systematic review. Journal of Child Neurology, 34: 237-47.

Meltzer MJ (2008) Brief report: sleep in parents of children with autism spectrum disorders. Journal of Pediatric Psychology, 33: 380-6.

Ming X, Sun YM, Nachajon RV, et al (2009) Prevalence of parasomnia in autistic children with sleep disorders. Pediatrics, 3: 1-10.

Morgan B, Nageye F, Masi G, et al (2020) Sleep in adults with autism spectrum disorder: a systematic review and meta-analysis of subjective and objective studies. Sleep Medicine, 65: 113-20.

Murray ML, Hsia Y, Glaser K, et al (2014) Pharmacological treatments prescribed to people with autism spectrum disorder (ASD) in primary health care. Psychopharmacology, 231: 1011-21.

National Institute for Health and Care Excellence (2012) Autism Spectrum Disorder in Adults: Diagnosis and Management (Clinical Guideline CG142). NICE.

National Institute for Health and Care Excellence (2015) Challenging Behaviour and Learning Disabilities: Prevention and Interventions for People with Learning Disabilities Whose Behaviour Challenges (NICE Guideline NG11). NICE.

National Institute for Health and Care Excellence (2017) Health App: Sleepio for Adults with Poor Sleep (Medtech Innovation Briefing MIB129). NICE.

National Institute for Health and Care Excellence (2018) Attention Deficit Hyperactivity Disorder: Diagnosis and Management (NICE Guideline NG87). NICE

Ohayon M (2002) Epidemiology of insomnia: what we know and what we still need to learn. Sleep Medicine Reviews, 6: 97-111.

Papadopoulos N, Sciberras E, Hiscock H, et al (2019) The efficacy of a brief behavioral sleep intervention in school-aged children with ADHD and comorbid autism spectrum disorder. Journal of Attention Disorders, 23: $341-50$.

Richdale AL, Schreck KA (2009) Sleep problems in autism spectrum disorders: prevalence, nature, and possible biopsychosocial aetiologies. Sleep Medicine Reviews, 13: 403-11.

Riemann D, Perlis ML (2009) The treatments of chronic insomnia: a review of benzodiazepine receptor agonists and psychological and behavioral therapies. Sleep Medicine Reviews, 13: 205-14.

Roberts CA, Smith KC, Sherman AK (2019) Comparison of online and faceto-face parent education for children with autism and sleep problems. Journal of Autism and Developmental Disorders, 49: 1410-22.
Saito M Hirota T, Sakamoto Y et al (2020) Prevalence and cumulative incidence of autism spectrum disorders and the patterns of co-occurring neurodevelopmental disorders in a total population sample of 5-yearsold children. Molecular Autism, 11(1): 35.

Scantlebury A, Mcdaid C, Dawson V, et al (2018) Non-pharmacological interventions for non-respiratory sleep disturbance in children with neurodisabilities: a systematic review. Developmental Medicine \& Child Neurology, 60: 1076-92.

Selsick H, O'Regan D (2018) Sleep disorders in psychiatry. BJPsych Advances, 24: 273-83

Shanahan P, Palod S, Smith K, et al (2019) Interventions for sleep difficulties in adults with an intellectual disability: a systematic review. Journal of Intellectual Disability Research, 63: 372-85.

Sivertsen B, Harvey A, Pallesen S, et al (2014) Mental health problems in adolescents with delayed sleep phase: results from a large populationbased study in Norway. Journal of Sleep Research, 24: 11-8.

Souders MC, Zavodny S, Eriksen W, et al (2017) Sleep in children with autism spectrum disorder. Current Psychiatry Reports, 19(6): 34.

Stein MA, Weiss M, Hlavaty L (2012) ADHD treatments, sleep, and sleep problems: complex associations. Neurotherapeutics, 9: 509-17.

Stores $G$ (2009) Aspects of parasomnias in childhood and adolescence. Archives of Disease in Childhood, 94: 63-9.

Stores G (2015) Sleep disorders in children and adolescents. BJPsych Advances, 21: 124-31.

Sung V, Hiscock H, Sciberras E, et al (2008) Sleep problems in children with attention-deficit/hyperactivity disorder. Archives of Pediatrics \& Adolescent Medicine 162: 336-42.

Takaesu Y, Inoue Y, Murakoshi A, et al (2016) Prevalence of circadian rhythm sleep-wake disorders and associated factors in euthymic patients with bipolar disorder. PLoS One, 11(7): e0159578.

Taylor DJ, Pruiksma KE (2014) Cognitive and behavioural therapy for insomnia (CBT-I) in psychiatric populations: a systematic review. International Review of Psychiatry, 26: 205-13.

Veatch OJ, Sutcliffe JS, Warren ZE, et al (2017) Shorter sleep duration is associated with social impairment and comorbidities in ASD. Autism Research, 10: 1221-38.

Wajszilber D, Santisteban J, Gruber R (2018) Sleep disorders in patients with ADHD: impact and management challenges. Nature and Science of Sleep, 10: 453-80.

Walker MP (2009) The role of sleep in cognition and emotion. Annals of the New York Academy of Sciences, 1156: 168-97.

Wiggs L, Stores G (2004) Sleep patterns and sleep disorders in children with autistic spectrum disorders: insights using parent report and actigraphy. Developmental Medicine and Child Neurology, 46: 372-80.

World Health Organization (1992) The ICD-10 classification of mental and behavioural disorders: Clinical Descriptions and Diagnostic Guidelines. WHO

Zablotsky B, Black LI, Maenner MJ, et al (2015) Estimated prevalence of autism and other developmental disabilities following questionnaire changes in the 2014 National Health Interview Survey. National Health Statistics Reports, (87): 1-20. 


\section{MCOs}

Select the single best option for each question:

1 The prevalence of sleep disorders in adults with autism spectrum disorder is:

a $20-50 \%$

b $30-60 \%$

c $40-70 \%$

d $50-80 \%$

e $60-90 \%$

2 As regards the statement that delaying treatment in adolescents with circadian rhythm sleep-wake disorders is acceptable as the sleep disorder will resolve in adulthood:

a this is true only for those with ADHD

b this is true only for those with ASD

c this is true only for those without ADHD and ASD

$d$ this is true

e this is false.
3 In advanced sleep-wake phase disorder, use of a light box with 10000 lux can be considered:

a never

b at $03.00 \mathrm{~h}$

c at $09.00 \mathrm{~h}$

$\mathrm{d}$ at $15.00 \mathrm{~h}$

e at $20.00 \mathrm{~h}$.

4 As regards the prevalence of insomnia in children and adults with ADHD:

a insomnia is not a reported sleep disorder in children with ADHD

b the prevalence in adults is lower than the prevalence in children

c the prevalence in adults is the same as the prevalence in children

$d$ the prevalence in adults is higher than the prevalence in children

e insomnia is not a reported sleep disorder in adults with $\mathrm{ADHD}$.
5 The STOP-Bang Questionnaire is a widely used screening tool for identifying:

a narcolepsy

b restless legs syndrome

c obstructive sleep apnoea

d parasomnias

e circadian rhythm sleep-wake disorders. 